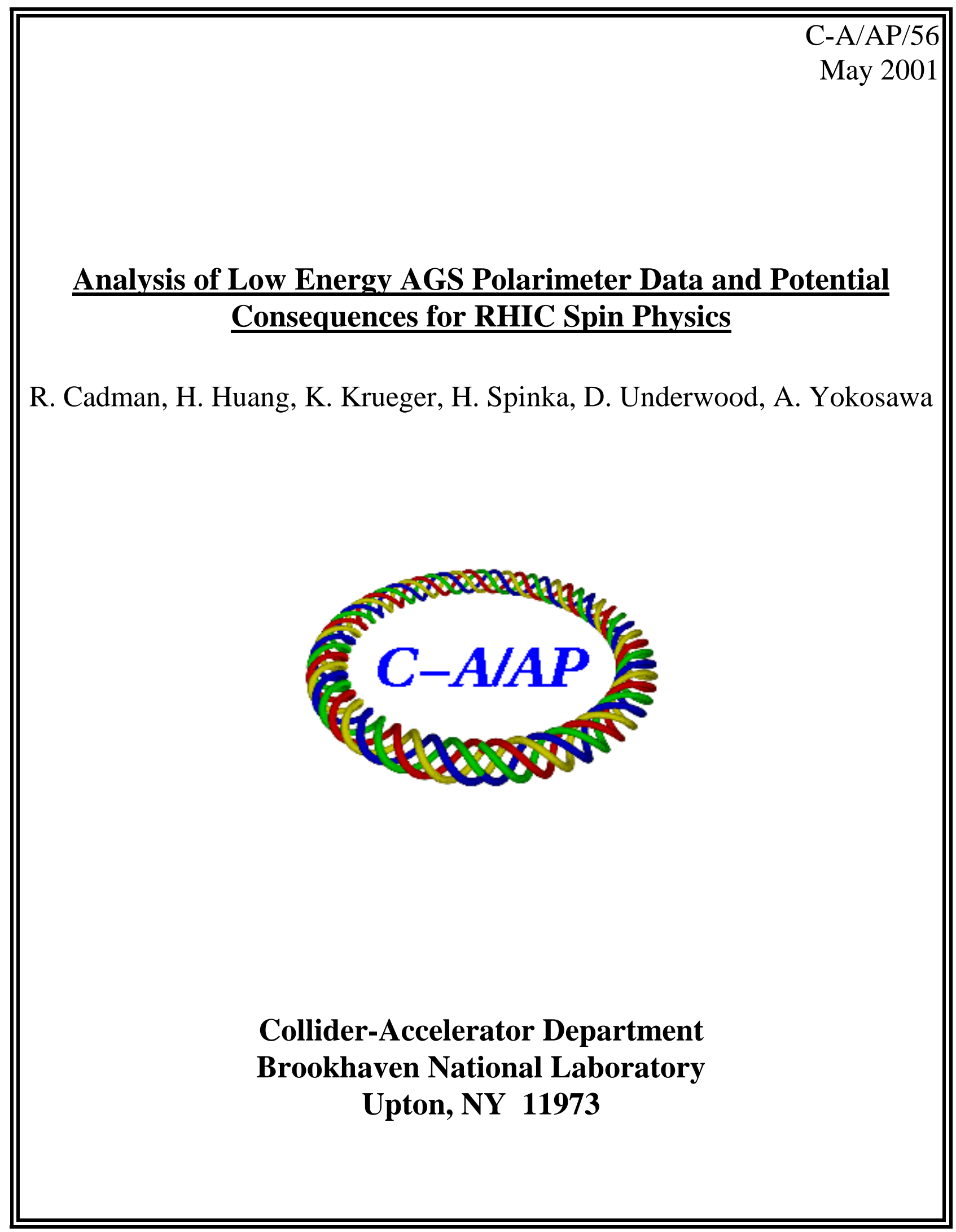




\title{
Analysis of Low Energy AGS Polarimeter Data and Potential Consequences for RHIC Spin Physics
}

\author{
R.Cadman,K.Krueger,H.Spinka, D.Underwood and A.Yokosawa \\ HEP Division,Argonne National Laboratory,Argonne,Ill.60439 \\ H.Huang \\ C-A Department,Brookhaven National Laboratory, Upton, NY11973
}

\begin{abstract}
The small asymmetries measured at $G \gamma=7.5$ during the RHIC spin commissioning were a serious concern. In earlier runs, asymmetries double those from the spin commissioning time (September 2000) had sometimes been observed, and there had been few changes to the AGS polarimeter hardware or operating conditions.

Recently, the observed changes in the asymmetries measured at $G \gamma=7.5$ have been ascribed to contamination of the carbon target asymmetry with that from the fishline target and vice-versa, because of the sizeable beam spot size compared to the separation of the targets. This note addresses this hypothesis using the observed asymmetries. This problem could directly impact spin physics at RHIC.
\end{abstract}

\section{INTRODUCTION}

Knowledge of absolute beam polarization is crucial for spin physics measurements. For RHIC, a Coulomb-Nuclear interference (CNI) polarimeter will operate in each ring. Their present calibrations depend on a chain of cross calibrations, calculations, and assumptions. 
Plans exist to perform an absolute beam polarization determination in a few years with a polarized gas target in RHIC. This measurement will provide a direct, accurate and simultaneous calibration of both CNI polarimeters.

Spin physics measurements before this calibration will continue to rely on the present results. However, the consistency of the chain of cross calibrations has been questioned by measurements with the AGS internal polarimeter slightly above the injection energy. The problem is that there is an inconsistency between past and recent measurements of the analyzing power in the AGS internal polarimeter as much as a factor of two! If this represents a true change in polarization, then it would mean an increase of a factor of four in the uncertainty of two-spin asymmetries, which would make some hoped for measurements meaningless!

The outline of this note is as follows. First, the measurements are described, as well as the assumptions. Next, the data with no fishline target are analyzed. This is followed by the results when both targets were present. Finally, conclusions are described.

\section{A. Some Background Information}

The data of interest were taken at $G \gamma=7.5$, where $G$ is the proton anomalous magnetic moment and $\gamma=E / m$ is the Lorentz factor. This corresponds to a laboratory momentum of $p=3.81 \mathrm{GeV} / \mathrm{c}$, whereas injection into the AGS occurs at $G \gamma=4.7$ or $p=2.27 \mathrm{GeV} / \mathrm{c}$. Injection into RHIC occurs at $G \gamma=46.5$ or $p=24.3 \mathrm{GeV} / \mathrm{c}$. The polarized beam originates in a polarized ion source. It then is accelerated through a radio-frequency quadrupole and LINAC to $200 \mathrm{MeV}$ kinetic energy and then through the Booster synchrotron into the AGS. The beam polarization is also measured at $200 \mathrm{MeV}$, but not in the Booster. [1]

Details of the AGS internal polarimeter have, unfortunately, never been published except in student dissertations, but an upcoming publication is expected to rectify that. Here we will just give a rudimentary description. The polarimeter consists of left and right arms

of scintillators placed at a nominal angle of $\sim 77.25^{\circ}$ from the beam line. There are two 
possible targets that are put into the beam during different running periods. One of the targets is pure carbon. The other target is fishline typically $C_{6} H_{11} N O$. They are both on the same target holder a distance of about 1" apart, but the target frame makes an angle of $30^{\circ}$ with the beam. The effective target separation perpendicular to the beam is $2.2 \mathrm{~cm}$.

The polarimeter measures the left-right asymmetry due to elastic scattering of a polarized proton beam off of the target. The asymmetry is defined by the following equation.

$$
\epsilon=\frac{\sqrt{N_{L}^{\uparrow} N_{R}^{\downarrow}}-\sqrt{N_{L}^{\downarrow} N_{R}^{\uparrow}}}{\sqrt{N_{L}^{\uparrow} N_{R}^{\downarrow}}+\sqrt{N_{L}^{\downarrow} N_{R}^{\uparrow}}}
$$

where $N_{L}^{\uparrow}$ is the number of scatters into the left counters with beam spin up and $N_{L}^{\downarrow}$ is the number of scatters into the left counter with beam spin down. $N_{R}^{\uparrow}$ and $N_{R}^{\downarrow}$ are defined in similar fashion.

\section{THE OBSERVATIONS}

The asymmetry in the AGS polarimeter has been measured in different years with both fishline and carbon targets at several different energies after acceleration up towards RHIC injection energy. This allowed the determination of the quasielastic background relative to the elastic scattering from the free hydrogen in the nylon. Then the effective anlyzing power of the nylon target can be calculated. Based on the measured ratio of asymmetries of nylon vs. carbon, one can get the effective analyzing power for the carbon target. This analyzing power encountered a challenge during RHIC polarized proton commissioning in September 2000, when a new source was first commissioned with 20 times higher intensity which was too high for the nylon target to survive. The measured carbon target asymmetry in 2000 was only about half what it was in 1999 even though the source polarization is believed to be only about $20 \%$ lower (0.84 vs. 0.68). A hypothesis has been made that the larger beam size at $G \gamma=7.5$ allowed cross-contamination of the results between the targets in 1999. However, data at $G \gamma=41.5$, where the beam spot is too small to strike both targets, shows the same factor of two difference between 1999 and $2000(2.02 \pm .35)$ if we use the highest carbon 
asymmetry achieved in Mar.1999! There are some that argue that the high value achieved in 1999 should be ignored in favor of a lower value that was achieved more consistently. In this case one gets a ratio between the years of $1.48 \pm 0.23$; this is the same as the ratio of the $200 \mathrm{MeV}$ polarization measurements between the years,1.37, within statistical errors.

Table I reports the $G \gamma=7.5$ measurements of maximum asymmetries, and one other case from the AGS polarimeter. These are taken from the logbooks. $\mathrm{P}(200 \mathrm{MeV})$ is the polarization measured before injection into the Booster and AGS. In general, this measurement was not close in time to the AGS results. Thus, there may have been some source polarization variation with time, though the magnitude of such variation is unknown. In the table $\epsilon_{f-o b s}$ is the observed value of proton scattering off of fishline, $\epsilon_{c-o b s}$ is the observed value of proton scattering off of carbon and $r_{o b s}=\epsilon_{f-o b s} / \epsilon_{c-o b s}$ is the observed value of the ratio.

To quantify the problem let us use the data in the table without any corrections which depend on our assumptions. If we use the 1997 data we can calculate an analyzing power for carbon $52.7 \times 10^{-3} / .786=.067$. If we use that to calculate the polarization in Sept.2000, we get $22.1 \times 10^{-3} / 0.067=.33$ and we would expect this to be decreased after acceleration and extraction into RHIC to give a polarization of $.33 \times .75=.25$. (How much it decreases depends on assumptions and simulations of the AGS.) The cause of such a low polarization at $G \gamma=7.5$ would not be understood, and could possibly occur again, jeopardizing future RHIC polarized proton running. ( This situtation would not be helped much even if the $\mathrm{P}(200 \mathrm{MeV})$ polarization increase from 0.65 to 0.80 .) 


\section{TABLES}

TABLE I. Asymmetries x $10^{-3}$ at $G \gamma=7.5$.

\begin{tabular}{cccccc}
\hline \hline $\mathrm{P}(200 \mathrm{MeV})$ & Date & $\epsilon_{f-o b s}$ & $\epsilon_{c-o b s}$ & $r_{o b s}$ & Notes \\
\hline .681 & July 1996 & $39.0 \pm 0.5$ & $25.8 \pm 0.4$ & $1.511 \pm 0.030$ & fishline out during carbon run \\
& & $23.0 \pm 0.5$ & $14.2 \pm 0.4$ & $1.597 \pm 0.056$ & fishline out during carbon run \\
.786 & July 1997 & $77.2 \pm 0.7$ & $52.7 \pm 1.1$ & $1.465 \pm 0.033$ & both carbon and fishline present \\
.842 & Mar 1999 & & $43.8 \pm 0.7$ & & " " \\
.65 & Sept. 2000 & & $22.1 \pm 0.8$ & & \\
& & & & \\
\hline \hline
\end{tabular}


It was also noted that in July 1996, some beam polarization may have been lost in the AGS before $G \gamma=7.5$ due to the snake magnet current setting. In addition, the fishline data in July 1996 were not collected close in time to the carbon results. In fact, the fishline target was broken when the carbon data were taken, as evident by a target sweep in position across the beam.

\section{SOME ASSUMPTIONS}

A number of assumptions will be made, and results derived. It will be shown that the data are not consistent with some subset of the assumptions. The consequences will be studied further in the end of this note.

Assumption 1: There is no beam depolarization between $200 \mathrm{MeV}$ and $G \gamma=7.5$ (3.81 $\mathrm{GeV} / \mathrm{c}$ ) in this time period except perhaps in July 1996. No cause of any such depolarization is known. The Booster operating conditions were studied to insure there was minimal depolarization in that machine in these runs.

Assumption 2: The beam polarization at $200 \mathrm{MeV}$ is stable with time. Measurments generally did not change very much within a given run period, though the $200 \mathrm{MeV}$ polarimeter operated only intermittently during each run.

Assumption 3: The AGS polarimeter operated reproducibly from year to year, and gave accurate results. However, at $G \gamma=7.5$, the beam spot was large and counts from the carbon target could contaminate the data from the fishline target and vice-versa.

Assumption 4: The beam profile will be assumed to be Gaussian, at least out to 3-4 $\sigma$. This will cover the separation of the carbon and fishline on the AGS polarimeter target holder.

Assumption 5: The carbon analyzing power for the AGS polarimeter, $A_{p C}$, can be taken from measurements at Saclay $(K . E .=2.8 G e V, p=3.62 G e V / c)$. Here $A_{p C}=0.033 \pm$ 0.002 (We have increased their reported error to include possible systematic errors). There are detailed differences in the Saclay polarimeter compared with the AGS polarimeter, in 
addition to the $19 \mathrm{GeV} / \mathrm{c}$ momentum difference. For example, the angles are slightly different $\left(73^{\circ}\right.$ vs. $\left.77^{\circ}\right)$, the angular acceptances, energy losses, and the degrader used in the AGS polarimeter are also different. Note that at $K E=2.4 \mathrm{GeV}(p=3.20 \mathrm{GeV})$ the Saclay result was substantially larger with $A_{p C}=0.045 \pm 0.003$.

Assumption 6: The ratio of the cross-sections of the fishline and carbon is not a strong function of energy.

\section{DATA WITH NO FISHLINE TARGET PRESENT}

With the previous assumptions and data, the AGS polarimeter analyzing powers can be computed in Sept. 2000 and July 1996. These are

1)Sept.2000 $A_{p C}=\epsilon_{c} / P(200 M e V)=0.0340 \pm 0.0020$ which is consistent with the Saclay result (Assumption 5).

2)July $1996 A_{p C} \geq 0.0379 \pm 0.00065$ which is consistent with the Saclay result. The inequality arises because of possible beam depolarization before $G \gamma=7.5$ in the AGS.

If it is further assumed that the equality holds in the case of the July 1996 data, then the two values of $A_{p C}$ differ by only $2 \sigma$. The weighted average of the two is then $\left\langle A_{p C}\right\rangle$ $=0.0375 \pm 0.00062$. This is already $2.2 \sigma$ away from the Saclay [2] value. Note that if the inequality holds, the disagreement becomes larger. Further conclusions will be discussed at the end of this note.

\section{DATA WITH BOTH TARGETS PRESENT - MARCH 1999}

The asymmetry measured in March 1999 is believed to be partly contaminated by the fishline target, so that what is observed can be expressed as a fractional sum of fishline and carbon asymmetries as follows:

$$
\epsilon_{o b s} \cong \frac{a \epsilon_{f}+b \epsilon_{c}}{(a+b)}
$$


Here $\epsilon_{c}$ and $\epsilon_{f}$ are the true values for the carbon and fishline asymmetries and $\epsilon_{\text {obs }}$ is the observed asymmetry. Note that the "square root" asymmetries used for the AGS polarimeter would have a very small systematic error for the fishline target asymmetry due to the offset target position. This acceptance difference is very small because to first order the acceptance cancels out of equation (1.1) and the target separation difference is small compared to the distance from the targets to the acceptance defining counters. It will be assumed that the carbon target was centered on the beamline and, as was just noted, small deviations from that will not affect the asymmetry.

We define a new quantity $\mathrm{R}=$ Rate(fishline)/Rate(carbon). This ratio depends on the relative thickness and density of the two targets and the relative cross-sections of fishline and carbon. The thickness of the carbon target was different in different years. Observations noted in the logbooks suggest that the ratio of counts from the fishline to carbon was $7.5 \pm 0.7$ for the same beam intensity on the target. This result was from a much higher beam energy, where there was little beam hitting the wrong target when centered on carbon or fishline. For the next calculation it will be assumed that the same ratio holds for $G \gamma=7.5$. Assign this ratio to be $R=7.5$.

The contamination factor will be $e^{-\left(s^{2}\right) /\left(2 \sigma^{2}\right)}$, where $\mathrm{s}=2.2 \mathrm{~cm}$ is the separation distance of the fishline and carbon targets perpendicular to the beam. Therefore, our equation becomes

$$
\epsilon_{c-o b s}=\frac{R e^{-s^{2} /\left(2 \sigma^{2}\right)} \epsilon_{f}+\epsilon_{c}}{R e^{-s^{2} /\left(2 \sigma^{2}\right)}+1} .
$$

We will define $r=\epsilon_{f} / \epsilon_{c}=A_{p f} / A_{p C}$. We wish to know the possible range of values that exist for $\sigma$ and r. These two parameters will then provide important constraints on $A_{p C}$ using the March 1999 data. If you examine figure 1, you will see that in the AGS energy range corresponding to $G \gamma=7.5$ to $46.5, r_{o b s}$, the observed value of $r$ varies from 1.5 to 3. However, the possibility of cross-contamination between targets at $G \gamma=7.5$ may have artifically reduced the value of $\mathrm{r}$ at that energy. Thus, a reasonable estimate of $\mathrm{r}$ would be 1.5 to 3.0. Note that with $A_{p C}=0.033$ from Saclay, $r=7.6$ would correspond to $A_{p f}=0.25$, 
or approximately the pp elastic scattering analyzing power. (For $A_{p p}$ we take the average of .31 [3] and .19 [4]. These data were taken at similar energies and angles.)

The beam spot size at $G \gamma=7.5$ can be estimated from target sweeps recorded in the logbooks. Unfortunately, none were shown at this energy during March 1999. The data from July 1996 and from Mei Bai's thesis (July 1997) show FWHM $600-800$ counts $0.6 "-0.8 "$, which implies $\sigma \simeq 0.75 \pm 0.10 \mathrm{~cm}$.

Data from the AGS IPM measurement of the beam size can also be used to estimate $\sigma$. These have been analyzed, and corrected for the difference in position between the beam monitors and the AGS polarimeter. This analysis suggests the following. In March 1999, close to the time the $G \gamma=7.5$ measurements were made, several IPM measurements were made giving a beam size of $\sigma=.72$ to $1.11 \mathrm{~cm}$. In July 1997, again close to the $G \gamma=7.5$ measurements, IPM measurements gave $\sigma=.77$ to $1.19 \mathrm{~cm}$. The maximum possible size at the AGS polarimeter for $G \gamma=7.5$ would be $\sigma \simeq 1.23 \mathrm{~cm}$, based on the injection energy $(G \gamma=4.7)$ and the limiting aperture in the AGS.

Returning to equation (5.2) we factor out $\epsilon_{c}$ and subsitute $\mathrm{r}$ to get

$$
\epsilon_{c-o b s}=\epsilon_{c} \frac{R r e^{-s^{2} / 2 \sigma^{2}}+1}{R e^{-s^{2} / 2 \sigma^{2}}+1}
$$

solving for $\mathrm{r}$ gives

$$
r=\frac{\epsilon_{c-o b s}}{\epsilon_{c}}\left[1+\frac{e^{s^{2} / 2 \sigma^{2}}}{R}\right]-\frac{e^{s^{2} / 2 \sigma^{2}}}{R} .
$$

Note $\mathrm{R}=7.5, \mathrm{~s}=2.2 \mathrm{~cm}$ and $\epsilon_{c-o b s}=0.0438 \pm 0.0007$. By putting in reasonable values of $\sigma$ and $\epsilon_{c}$ into the equations we can see what range of values are possible for $\mathrm{r}$. Plots of $\mathrm{r}$ vs. $\epsilon_{c}$ are shown in figure 2, for various values of the beam width. [The above equation is just reminding us that a given $\epsilon_{c-o b s}$ can be produced by different pairs of $\sigma$ and $\epsilon_{c}$.]

The value of $\mathrm{r}$ computed above is the true value of $\mathrm{r}$. Our measured value, $r_{o b s}=$ $\epsilon_{f-o b s} / \epsilon_{c-o b s}$, will be less than the true value. We will derive an equation for $r_{o b s}$ below.

Similar to equation (5.3) for $\epsilon_{c-o b s}$ we have

$$
\epsilon_{f-o b s}=\frac{R \epsilon_{f}+e^{-s^{2} / 2 \sigma^{2}} \epsilon_{c}}{R+e^{-s^{2} / 2 \sigma^{2}}}
$$


dividing equation (5.5) by (5.3), we get

$$
r_{o b s}=\frac{R \epsilon_{f}+e^{-s^{2} / 2 \sigma^{2}} \epsilon_{c}}{\epsilon_{c}+R e^{-s^{2} / 2 \sigma^{2}} \epsilon_{f}}\left(\frac{y}{x}\right)
$$

where $y=1+R e^{-s^{2} / 2 \sigma^{2}}$ and $x=R+e^{-s^{2} / 2 \sigma^{2}}$.

This formula is used to plot the value of $r_{o b s}$ you would expect to measure in figure 3 , ( $r_{\text {obs }}$ is always less than the true value.) The difference between the measured value of $\mathrm{r}$ and the true value of $\mathrm{r}$ increases as $\epsilon_{c-o b s}$ diverges from its true value, as you would expect.

The plot shows that as the beam spot decreases, the value of $r=\epsilon_{f} / \epsilon_{c}=A_{p f} / A_{p C}$ increases quite rapidly for $\sigma$ less than about $0.85 \mathrm{~cm}$. Furthermore, unless the beam spot size was larger than $\sigma \sim 1.0 \mathrm{~cm}, \mathrm{r}$ becomes too large even for $\epsilon_{c}=0.033$. With the Saclay value of $A_{p C}=0.033$, this would correspond to $100 \%$ beam polarization. Note that results of the IPM analysis allow for larger $\sigma$ (up to $\sim 1.1 \mathrm{~cm})$.

\section{LIMITING CASES OF OUR EQUATIONS}

It is instructive to look at a limiting case of a very wide beam by letting $\sigma \rightarrow \infty$. Take equation (5.5) and let the beam size go to infinity to get

$$
\lim _{\sigma \rightarrow \infty} \epsilon_{f-o b s}=\frac{\epsilon_{f}+\frac{1}{R} \epsilon_{c}}{1+\frac{1}{R}} .
$$

Now substitute $\epsilon_{c}=\epsilon_{f} / r$ to get

$$
\lim _{\sigma \rightarrow \infty} \epsilon_{f-o b s}=\epsilon_{f} \frac{R+\frac{1}{r}}{R+1}
$$

As long as $r$ is greater than one, which is without any doubt true, then $\epsilon_{f-o b s}$ is always less than the true value. For $r=2$ and $R=7.5, \epsilon_{f-o b s}=\frac{16}{17} \epsilon_{f}$. As the beam becomes narrower ( $\sigma$ becomes smaller) the observed value of $\epsilon_{f-o b s}$ becomes closer to its true value.

$$
\lim _{\sigma \rightarrow 0} \epsilon_{f-o b s}=\epsilon_{f}
$$

Look at carbon for this same limiting case and we get the following. 


$$
\lim _{\sigma \rightarrow \infty} \epsilon_{c-o b s}=\frac{\epsilon_{c}+R \epsilon_{f}}{1+R}
$$

substitution for $\epsilon_{f}$ gives

$$
\lim _{\sigma \rightarrow \infty} \epsilon_{c-o b s}=\epsilon_{c} \frac{1+r R}{1+R}
$$

So as long as $\mathrm{r}$ is greater than one, $\epsilon_{c-o b s}$ is always greater than its true value. Using the same values of $R$ and $r$ we get

$$
\epsilon_{c-o b s}=\frac{32}{17} \epsilon_{c}
$$

Combining the results into the equation for $r_{o b s}$ gives

$$
\lim _{\sigma \rightarrow \infty} r_{o b s}=\lim _{\sigma \rightarrow \infty} \frac{\epsilon_{f-o b s}}{\epsilon_{c-o b s}}=1
$$

identically, regardless of the value of $\mathrm{r}$ and $\mathrm{R}$. This result is not surprising because the beam intensity in this extreme case does not vary over the targets no matter where we place the targets, so you would always measure the same asymmetry whether you centered the beam on the fishline or you centered it on the carbon.

We conclude that the measured value of the fishline asymmetry should always be close to the true value and never greater than the true value. The measured carbon asymmetry could approach the true value of the fishline asymmetry under extreme and unlikely conditions, but the measured value of $r, r_{o b s}$, would approach 1 and this was never observed.

\section{DATA WITH BOTH TARGETS PRESENT - JULY 1997}

In this case, both "fishline" and "carbon" target asymmetries are measured very close in time, in the pattern fishline - carbon - fishline (runs 1316-1318). The number of events for the three runs were essentially equal, and the number of spills to achieve these events were 52, 85 and 49 respectively. Thus to a good approximation $R_{o b s} \sim \frac{85}{50} \sim 1.70$. R is

different from 1999 because the carbon target is different. (Another estimate for R done on higher energy target sweeps gives $R=2.42 \pm .32$. This is within statistical error of our 
low energy estimate. Also, note that 1.70 is an observed value and later we will correct for cross-contamination.)

The measurements are

$$
\begin{gathered}
\epsilon_{f-o b s}=0.0772 \pm 0.0007=\epsilon_{f} \frac{R}{R+e^{-s^{2} / 2 \sigma^{2}}}+\epsilon_{c} \frac{e^{-s^{2} / 2 \sigma^{2}}}{R+e^{-s^{2} / 2 \sigma^{2}}} \\
\epsilon_{c-o b s}=0.0527 \pm 0.0001=\epsilon_{f} \frac{R e^{-s^{2} / 2 \sigma^{2}}}{R e^{-s^{2} / 2 \sigma^{2}}+1}+\epsilon_{c} \frac{1}{R e^{-s^{2} / 2 \sigma^{2}}+1}
\end{gathered}
$$

or if we divide through by the $200 \mathrm{MeV}$ beam polarization $(0.786 \pm 0.005)$, we get

$$
\begin{aligned}
& A_{p f-o b s}=0.0982 \pm 0.0011=A_{p C} \frac{r R+e^{-s^{2} / 2 \sigma^{2}}}{R+e^{-s^{2} / 2 \sigma^{2}}} \\
& A_{p c-o b s}=0.0670 \pm 0.0015=A_{p C} \frac{r R e^{-s^{2} / 2 \sigma^{2}}+1}{R e^{-s^{2} / 2 \sigma^{2}}+1}
\end{aligned}
$$

Taking the ratios cancels out $A_{p C}$ and we get

$$
r_{o b s}=\frac{A_{p f-o b s}}{A_{p c-o b s}}=1.465 \pm 0.033=\left(\frac{r R+e^{-s^{2} / 2 \sigma^{2}}}{r R e^{-s^{2} / 2 \sigma^{2}}+1}\right)\left(\frac{R e^{-s^{2} / 2 \sigma^{2}}+1}{R+e^{-s^{2} / 2 \sigma^{2}}}\right)
$$

Simplifying this expression gives

$$
r=\left(\frac{1}{R}\right) \frac{e^{-s^{2} / 2 \sigma^{2}}\left(R e^{-s^{2} / 2 \sigma^{2}}+1\right)-\left(A_{p f-o b s} / A_{p c-o b s}\right)\left(R+e^{-s^{2} / 2 \sigma^{2}}\right)}{e^{-s^{2} / 2 \sigma^{2}}\left(A_{p f-o b s} / A_{p c-o b s}\right)\left(R+e^{-s^{2} / 2 \sigma^{2}}\right)-\left(R e^{-s^{2} / 2 \sigma^{2}}+1\right)} .
$$

We can also correct the measured value of $R$ by using the following equation:

$$
R=\frac{R_{o b s}-e^{-s^{2} / 2 \sigma^{2}}}{1-R_{o b s} e^{-s^{2} / 2 \sigma^{2}}}
$$

Then the following table applies. Figure 4 shows a plot of the variables from Table II.

TABLE II. Range of possible values of $A_{p C}$

\begin{tabular}{lccccc}
\hline \hline$\sigma(\mathrm{cm})$ & 0.75 & 0.85 & 0.95 & 1.05 & 1.25 \\
$e^{-s^{2} / 2 \sigma^{2}}$ & 0.0135 & 0.0351 & 0.0685 & 0.1114 & 0.2125 \\
$R$ & 1.73 & 1.77 & 1.85 & 1.95 & 2.33 \\
$\mathrm{r}$ & 1.48 & 1.52 & 1.58 & 1.68 & 2.08 \\
$A_{p C}$ & 0.066 & 0.065 & 0.063 & 0.060 & 0.049 \\
\hline \hline
\end{tabular}


It was estimated that $\sigma \sim 0.77$ to $1.19 \mathrm{~cm}$ for these runs, but the derived values for $\mathrm{r}$ and $A_{p C}$ are only weakly dependent on $\sigma$, as can be seen in the table. Furthermore the value of $A_{p C}$ is much larger than the Saclay value by about a factor of 2 .

\section{BEAM NOT CENTERED ON A TARGET}

An unspoken assumption when deriving the above equations was that the beam was centered on one of the targets. One could get $A_{p C}$ to match the Saclay value if the beam is centered somewhere between the two targets. Figure 5 shows $A_{p C}$ as a function of target position. The beam size was taken from the target scan as $\sigma=0.82 \mathrm{~cm}$. As an example, for $A_{p C}=0.034$, the beam should be centered at 2760 instead of 3100, approximately one-third of the way between the targets $\left(\sim 0.34^{\prime \prime} \cos \left(30^{\circ}\right) \sim 0.75 \mathrm{~cm}\right)$. For the analysis shown in figure 5 , the equations change to the following form.

$$
\begin{gathered}
\epsilon_{f-o b s}=\epsilon_{f} \frac{R e^{-x_{o}^{2} / 2 \sigma^{2}}}{R e^{-x_{o}^{2} / 2 \sigma^{2}}+e^{-\left(s+x_{o}\right)^{2} / 2 \sigma^{2}}}+\epsilon_{c} \frac{e^{-\left(s+x_{o}\right)^{2} / 2 \sigma^{2}}}{R e^{-x_{o}^{2} / 2 \sigma^{2}}+e^{-\left(s+x_{o}\right)^{2} / 2 \sigma^{2}}} \\
\epsilon_{c-o b s}=\epsilon_{f} \frac{R e^{-\left(s-x_{o}\right)^{2} / 2 \sigma^{2}}}{R e^{-\left(s-x_{o}\right)^{2} / 2 \sigma^{2}}+e^{-x_{o}^{2} / 2 \sigma^{2}}}+\epsilon_{c} \frac{e^{-x_{o}^{2} / 2 \sigma^{2}}}{R e^{-\left(s-x_{o}\right)^{2} / 2 \sigma^{2}}+e^{-x_{o}^{2} / 2 \sigma^{2}}} \\
R=\frac{R_{o b s}-e^{-s^{2} / 2 \sigma^{2}} e^{-2 s x_{o} / \sigma^{2}}}{1-R_{o b s} e^{-s^{2} / 2 \sigma^{2}} e^{+2 s x_{o} / \sigma^{2}}}
\end{gathered}
$$

Remembering that $r_{o b s}=A_{p f-o b s} / A_{p c-o b s}$ we get the following equation to replace (7.6)

$$
r=\left(\frac{1}{R}\right) \frac{e^{-s^{2} / 2 \sigma^{2}}\left(R e^{-s^{2} / 2 \sigma^{2}}+e^{-2 s x_{o} / 2 \sigma^{2}}\right)-r_{o b s}\left(R+e^{-s^{2} / 2 \sigma^{2}} e^{-2 s x_{o} / 2 \sigma^{2}}\right)}{r_{o b s} e^{-s^{2} / 2 \sigma^{2}}\left(R e^{+2 s x_{o} / 2 \sigma^{2}}+e^{-s^{2} / 2 \sigma^{2}}\right)-\left(R e^{-s^{2} / 2 \sigma^{2}} e^{+2 s x_{o} / 2 \sigma^{2}}+1\right)} .
$$

Where $x_{o}$ is the offset of the beam position between the targets.

\section{SUMMARY}

The available AGS polarimeter data at $G \gamma=7.5$ were analyzed under a number of assumptions, as given in section B. The results were: Consistent Results 
- The July 1996 and September 2000 values for $A_{p C}$ were consistent, but the average was $\geq 2.2 \sigma$ away from the Saclay result. (Including possible systematic error in the Saclay result.)

- The March 1999 results are consistent with the assumptions if the beam width was $\sigma \geq 1.0 \mathrm{~cm}$ when $\epsilon_{c-o b s}$ was measured.

\section{Inconsistent Results}

- The July 1997 data are inconsistent with the assumptions, with a derived $A_{p C}$ about double the Saclay result, unless the beam was centered between the targets for most of the running period and the beam was wide. Furthermore, the ratio $A_{p f} / A_{p C}$ is close to 1.5 , which could constrain the March 1999 values if $\sigma$ were better known.

- Figure 6 combines all the carbon data in a common plot of $r=A_{p f} / A_{p C}$ as a function of $A_{p C}$. The September 2000 measurement constrains $A_{p C}$, but not r or $A_{p f}$. The July 1996 data is similar, but only provides a lower limit on $A_{p C}$. The March 1999 result is a band of values, bounded by a curve for $\sigma=0.75 \mathrm{~cm}$, the smallest beam size, and $\sigma=1.25 \mathrm{~cm}$, the largest beam size expected. Finally, the July 1997 points yield a narrow band of allowed values, depending on the beam size. It can be seen from the figure that the September 2000 and July 1997 results are inconsistent, there being no overlapping value for $A_{p C}$.

If we relax the requirement that the beam was centered on the targets, as in Section VII, "agreement" can be achieved under extreme conditions. Figure 7 shows the same data as fig.6 with additional points for July 1997 with beam offsets of $\left(x_{o}\right) 2.5$ and $5.0 \mathrm{~mm}$. Thus, consistency can be achieved if the beam was offset at least $5 \mathrm{~mm}$ from the target and had the maximum allowed beam size. This seems highly unlikely.

- Using the mechanism of cross-contamination between targets to explain the different values of $\epsilon$ can not under any circumstance explain the high value of $\epsilon_{f}$ seen in July 1997. This mechanism would serve only to decrease $\epsilon_{f}$. 


\section{CONCLUSIONS}

Based on all this evidence we believe that either a)the AGS polarimeter did not operate reproducibly from run period to run period, or b)there is depolarization between the 200 $\mathrm{MeV}$ polarimeter and the AGS polarimeter, and the Saclay $A_{p C}$ value does not apply to the AGS polarimeter ( different operating conditions) or c)both of these are true!

Finally, we conclude that there are enough doubts and questions about the measured asymmetries (also it is too important to the future of spin physics at RHIC) that more experimentation is needed. We need to repeat measurements at $G \gamma=7.5$ with both targets

present. First, we can see if we get the same results with both targets in as we did in July 1997 or September 1999. Second, we can move the beam accross the targets and measure the asymmetry at different positions to see if it varies in a manner consistent with the equations derived here. We also need to install forward arms in the AGS polarimeter, in order to measure pp elastic scattering directly.

The actual cause of the different asymmetries seen in different years could be entirely instrumental, or partly instrumental and partly a real change of polarization, or entirely a real change of polarization. The worst case scenario, if we fail to resolve this question, is that the beam polarization during RHIC running will be only half what was expected and the RHIC data will be compromised. 


\section{REFERENCES}

[1] H.Huang,et.al., Phys.Rev.Let.,73,2982(1994)

[2] J.Bystricky,et.al., Let.Nuo.Cim.,41,285(1984)

[3] F.Perrot,et.al.,Nuc.Phys.B,294,1001(1987)

[4] P.Grannis,et.al.,Phys.Rev.,148,148(1966) 


\section{FIGURES}

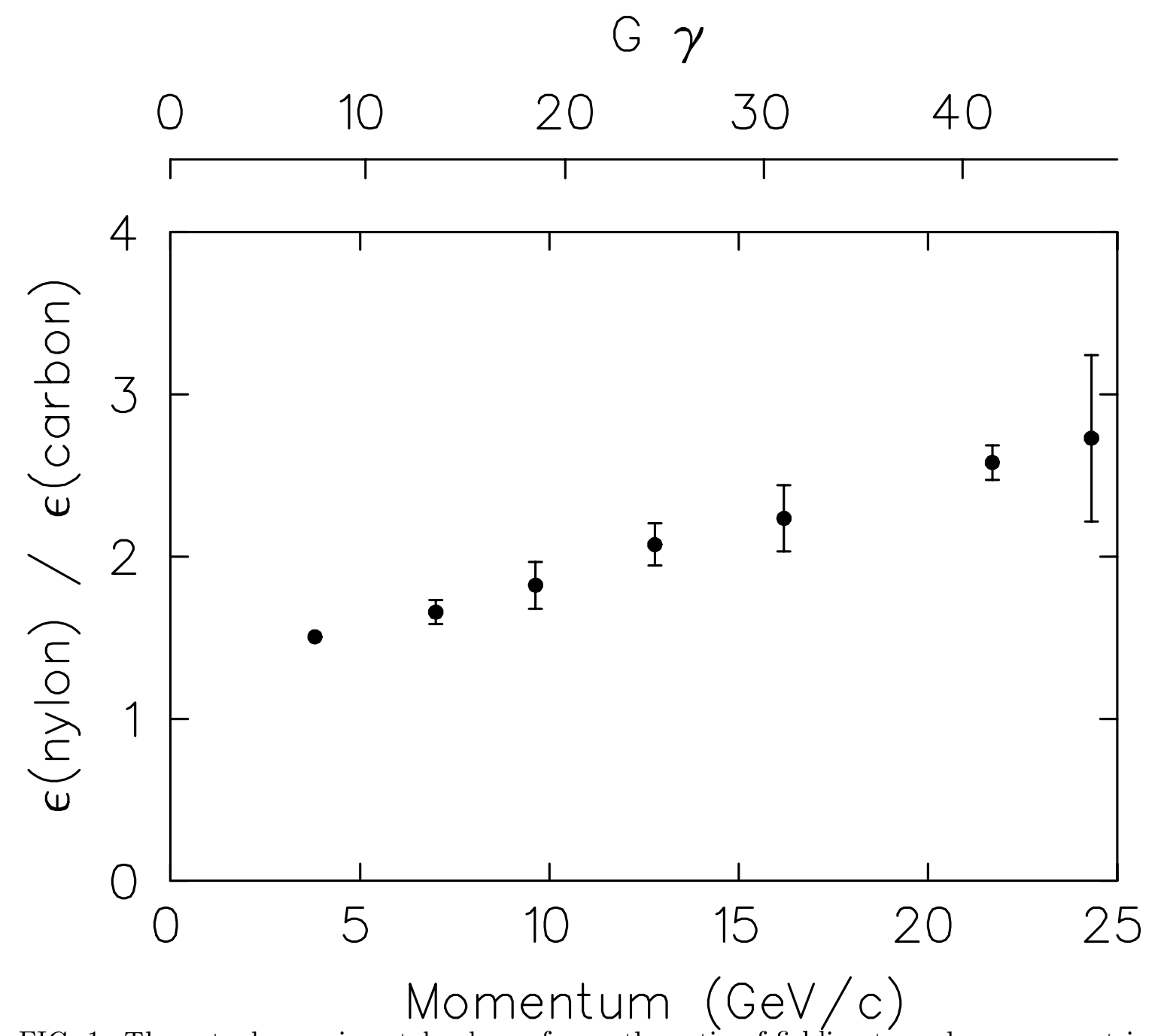

FIG. 1. The actual experimental values of $r_{o b s}$, the ratio of fishline to carbon asymmetries as a function of momentum. $p=3.8 \mathrm{GeV} / \mathrm{c}$ corresponds to $G \gamma=7.5$. 


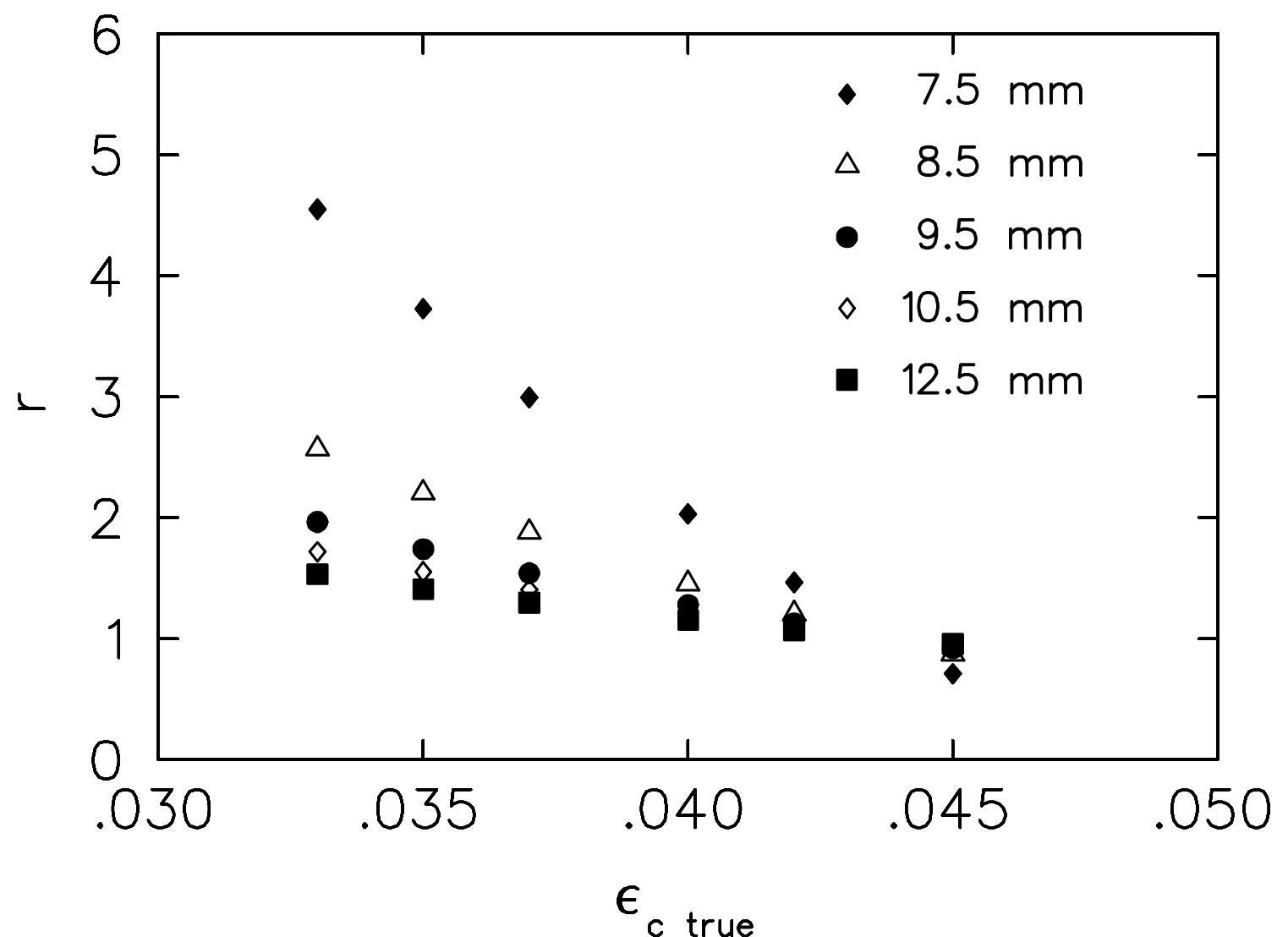

FIG. 2. The true value of $r$ plotted against the true value of carbon asymmetry for different beam widths. All curves intersect at $\mathrm{r}=1, \epsilon=.0438$. 


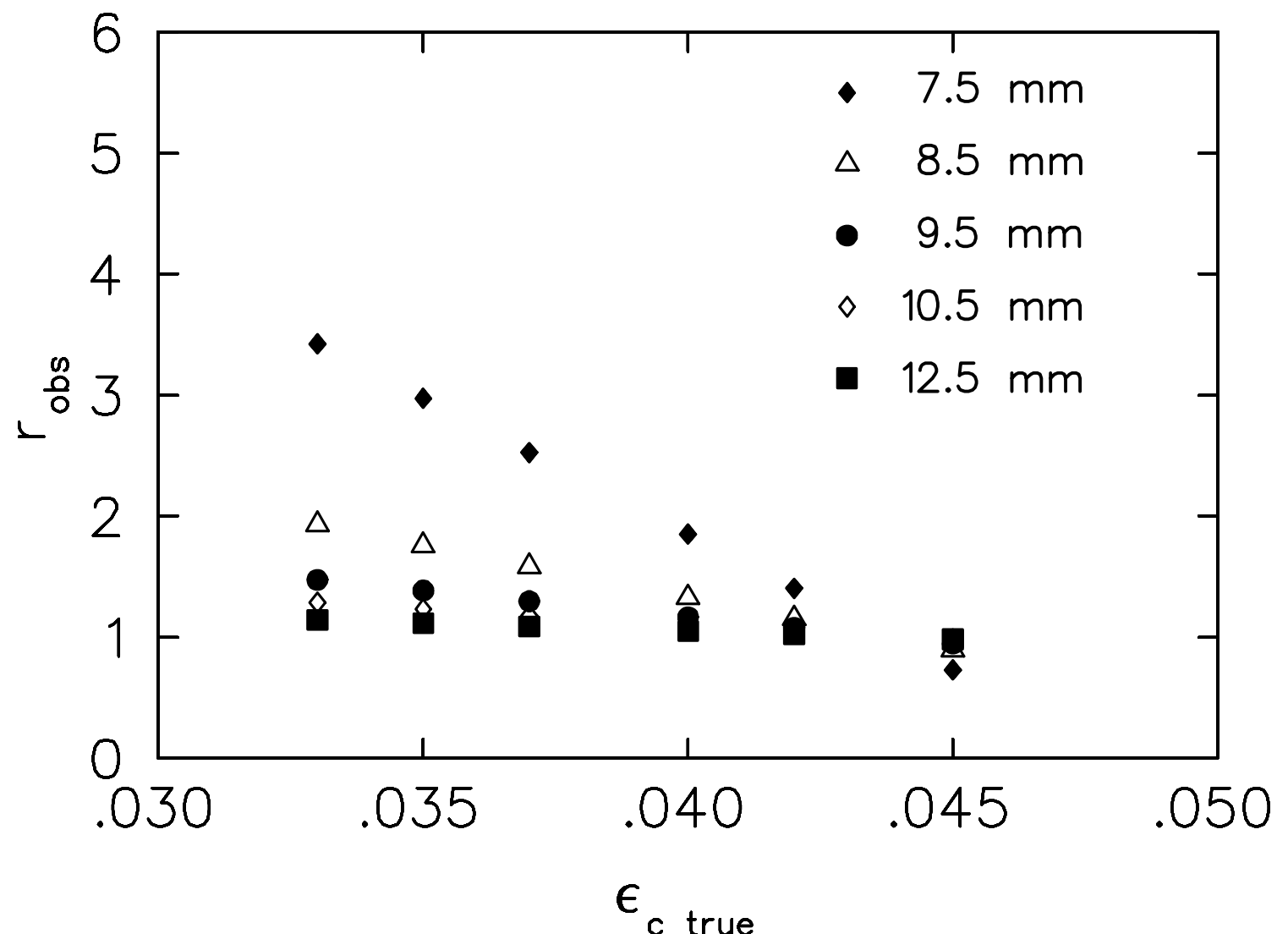

FIG. 3. The value of $\mathrm{r}$ expected to be observed against the true value of carbon asymmetry for different beam widths. 

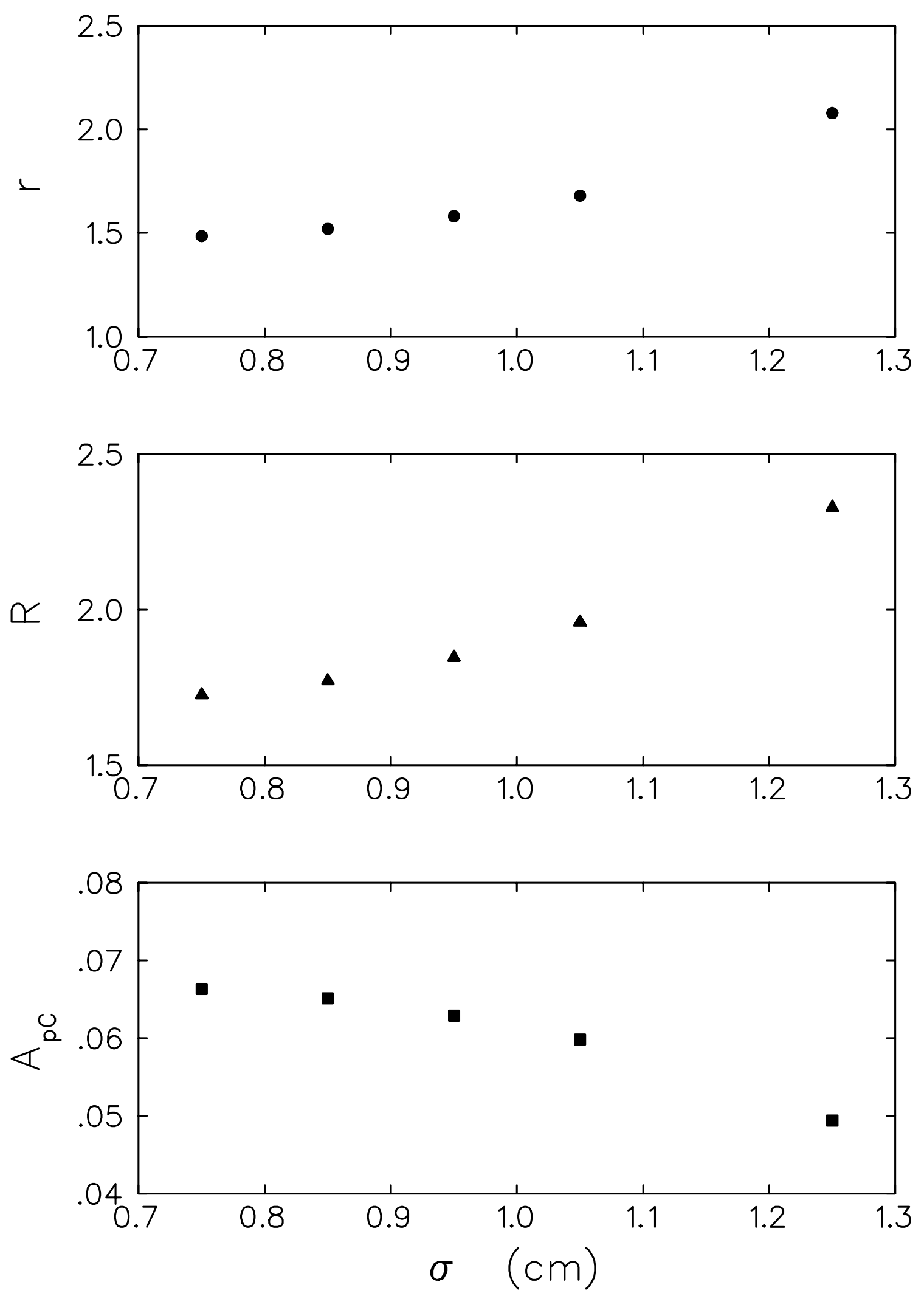

FIG. 4. quantities vs.beam width 


\section{asymmetry ratio vs. carbon target position}

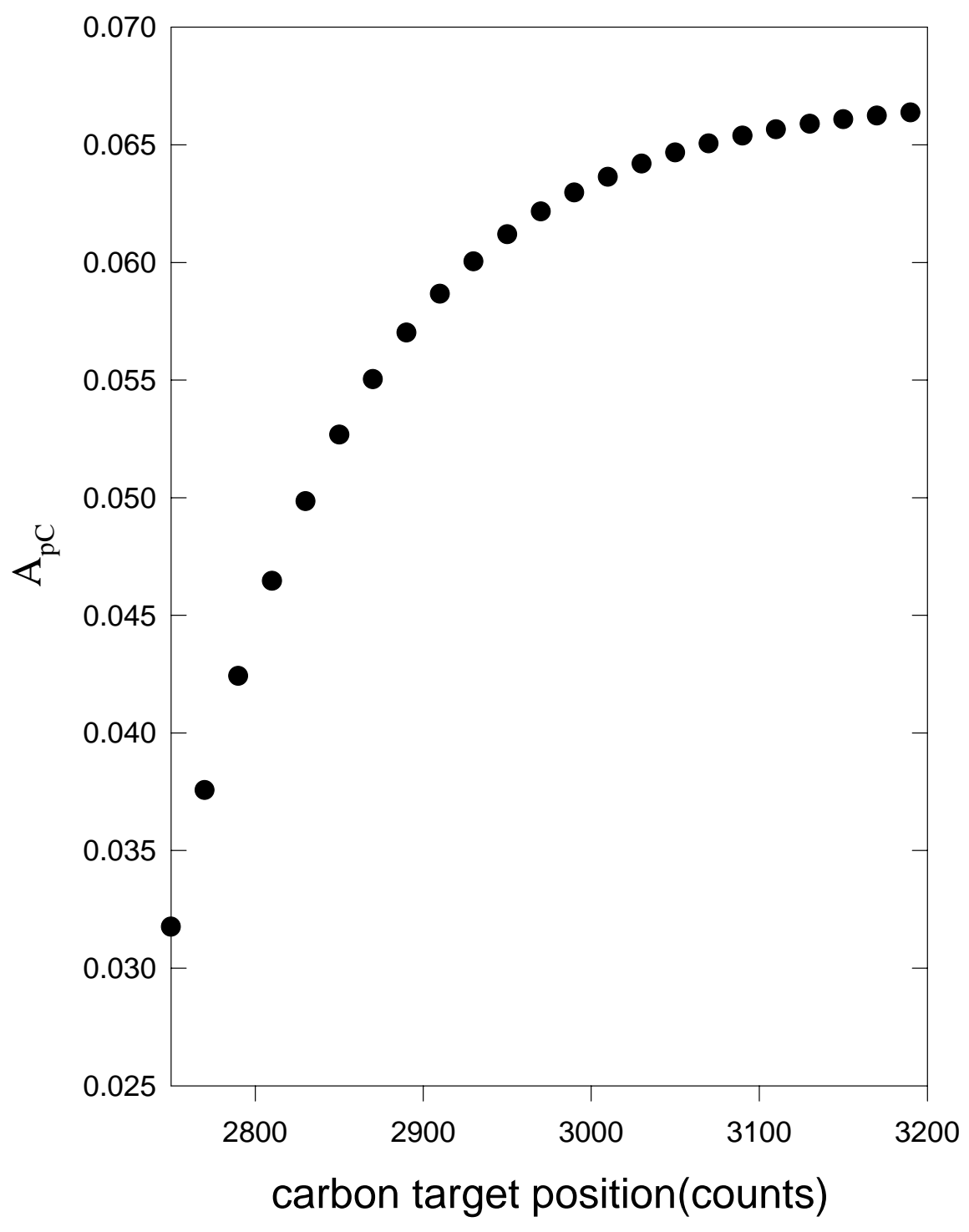

FIG. 5. $A_{p C}$ vs beam position. The targets are $1000 \operatorname{counts}(=1.0$ ") apart. The nominal position of the carbon target is at 3100 . An offset of the beam by $\sim 340$ counts or 0.34 " would yield the Saclay value for $A_{p C}$ 


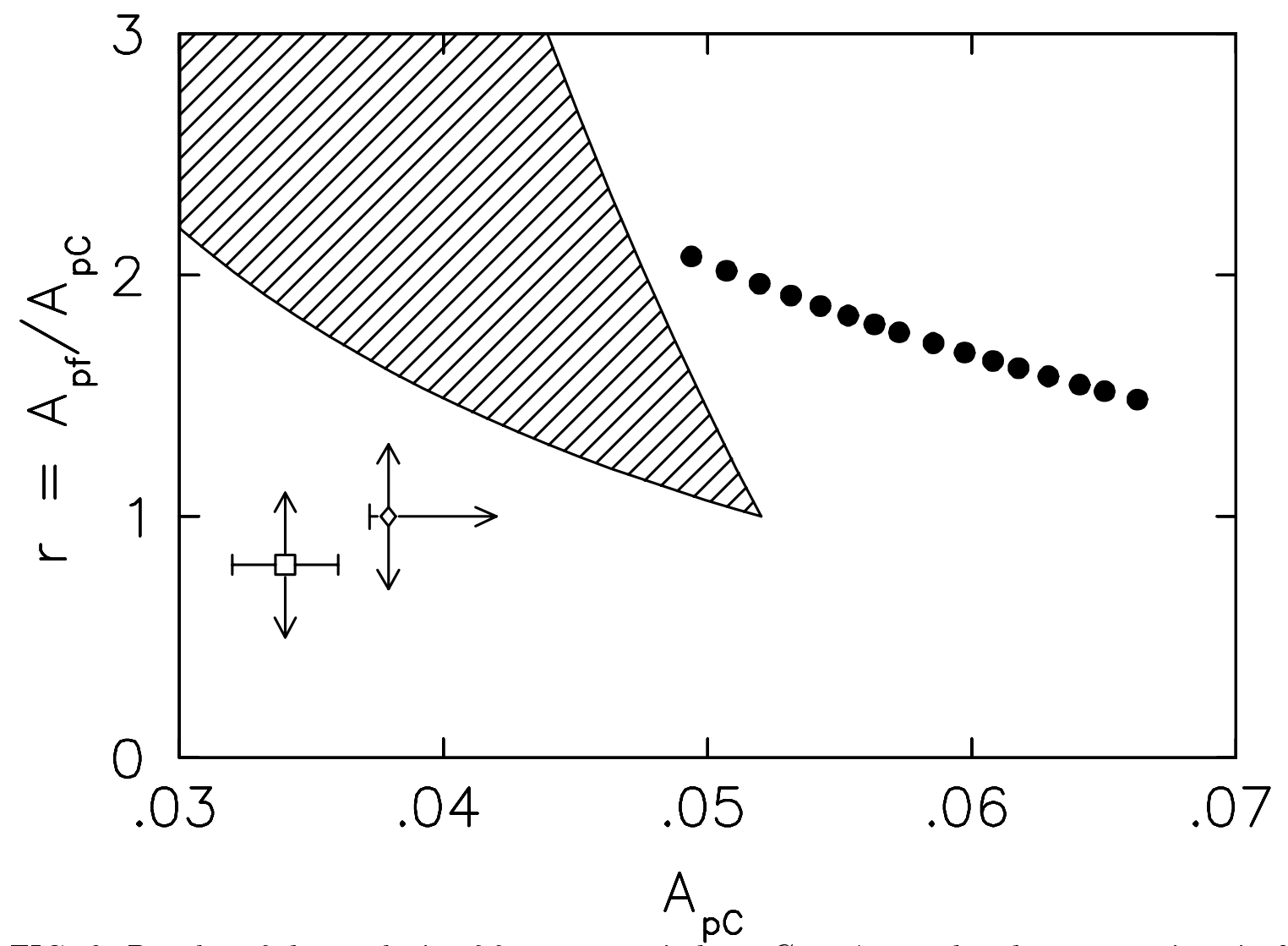

FIG. 6. Results of the analysis of four run periods at $G \gamma=7.5$ under the assumptions in Sec. III. The data for Sept.2000 ( $\square)$ and July $1996(\diamond)$ provide values for $A_{p C}$, but not for $A_{p f}$ or $\mathrm{r}$. The March 1999 results at small beam size $(\sigma=7.5)$ and large beam size $(\sigma=12.5 \mathrm{~mm})$ bound a region of acceptable values for $\mathrm{r}$ and $A_{p C}$ marked off by diagonal lines above. The July 1997 $\operatorname{data}(\bullet)$ give a narrow band of values, where the full range of allowable beam size, from $\sigma=7.5$ to $12.5 \mathrm{~mm}$, are shown. No value of $A_{p C}$ is consistent for all four run periods. 


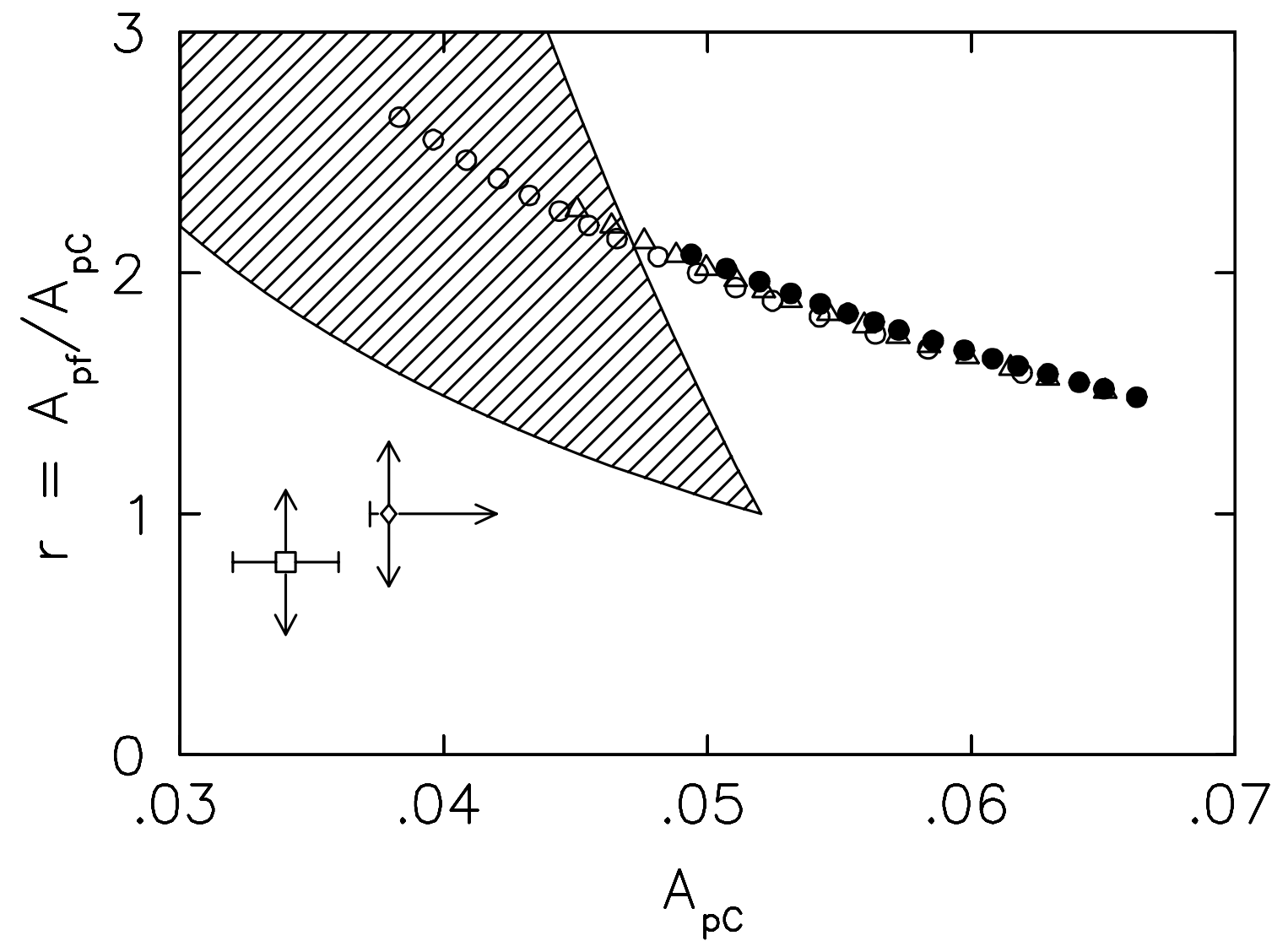

FIG. 7. All the same data from fig.6 is plotted here plus points for July 1997 have been added assuming different offsets of the beam from the target $(0,5.0 \mathrm{~mm}$, and $\triangle, 2.5 \mathrm{~mm})$. 